Supporting information for:

\title{
Validation and evaluation of High-resolution Orbitrap mass spectrometry on molecular characterization of dissolved organic matter (DOM)
}

Qiong Pan, Xiaocun Zhuo, Chen He*, Yahe Zhang, Quan Shi*

State Key Laboratory of Heavy Oil Processing, Petroleum Molecular Engineering Center (PMEC), China University of Petroleum, Beijing 102249, China

Contents

(1) Table S1 Instrumental parameter of ESI-Orbitrap MS.

(2) Figure S1 Mass distribution of condensate oil spectra of FT-ICR MS and Orbitrap MS.

(3) Figure S2 Distribution of O10 compounds under different ion spray voltage.

(4) Figure S3 Expanded spectra under evaluated ion spray voltage at $\mathrm{m} / \mathrm{z} 351$.

(5) Figure S4 Distribution of $\mathrm{O} 10$ compounds under $275^{\circ} \mathrm{C}$ (left), $300^{\circ} \mathrm{C}$ (right) ion transfer tube temperature.

(6) Figure S5 Distribution of O10 compounds under 20\% (left), 100\% (right) rf lens.

(7) Figure S6 Typical category of compounds in different area of van Krevelen diagram. 
Table S1. Instrumental parameter of ESI-Orbitrap MS

\begin{tabular}{|c|c|c|c|c|c|c|c|}
\hline Group & Sequence & $\begin{array}{c}\text { Neg ion } \\
\text { spray voltage }\end{array}$ & $\begin{array}{l}\text { sheath } \\
\text { gas }\end{array}$ & aux gas & $\begin{array}{l}\text { ion transfer } \\
\text { tube temp }\end{array}$ & rf lens & AGC target \\
\hline A & 1 & 2600 & 10 & 2 & 300 & 60 & 500000 \\
\hline A & 2 & 2700 & 10 & 2 & 300 & 60 & 500000 \\
\hline A & 3 & 2800 & 10 & 2 & 300 & 60 & 500000 \\
\hline A & 4 & 2900 & 10 & 2 & 300 & 60 & 500000 \\
\hline A & 5 & 3000 & 10 & 2 & 300 & 60 & 500000 \\
\hline A & 6 & 3100 & 10 & 2 & 300 & 60 & 500000 \\
\hline $\mathrm{B}$ & 1 & 2600 & 5 & 2 & 300 & 60 & 500000 \\
\hline B & 2 & 2600 & 7 & 2 & 300 & 60 & 500000 \\
\hline $\mathrm{B}$ & 3 & 2600 & 9 & 2 & 300 & 60 & 500000 \\
\hline $\mathrm{B}$ & 4 & 2600 & 11 & 2 & 300 & 60 & 500000 \\
\hline $\mathrm{B}$ & 5 & 2600 & 13 & 2 & 300 & 60 & 500000 \\
\hline B & 6 & 2600 & 15 & 2 & 300 & 60 & 500000 \\
\hline $\mathrm{C}$ & 1 & 2600 & 10 & 1 & 300 & 60 & 500000 \\
\hline $\mathrm{C}$ & 2 & 2600 & 10 & 2 & 300 & 60 & 500000 \\
\hline $\mathrm{C}$ & 3 & 2600 & 10 & 4 & 300 & 60 & 500000 \\
\hline $\mathrm{C}$ & 4 & 2600 & 10 & 6 & 300 & 60 & 500000 \\
\hline $\mathrm{D}$ & 1 & 2600 & 10 & 2 & 200 & 60 & 500000 \\
\hline $\mathrm{D}$ & 2 & 2600 & 10 & 2 & 225 & 60 & 500000 \\
\hline $\mathrm{D}$ & 3 & 2600 & 10 & 2 & 250 & 60 & 500000 \\
\hline $\mathrm{D}$ & 4 & 2600 & 10 & 2 & 275 & 60 & 500000 \\
\hline $\mathrm{D}$ & 5 & 2600 & 10 & 2 & 300 & 60 & 500000 \\
\hline E & 1 & 2600 & 10 & 2 & 300 & 20 & 500000 \\
\hline $\mathrm{E}$ & 2 & 2600 & 10 & 2 & 300 & 40 & 500000 \\
\hline E & 3 & 2600 & 10 & 2 & 300 & 60 & 500000 \\
\hline $\mathrm{E}$ & 4 & 2600 & 10 & 2 & 300 & 80 & 500000 \\
\hline $\mathrm{E}$ & 5 & 2600 & 10 & 2 & 300 & 100 & 500000 \\
\hline $\mathrm{E}$ & 6 & 2600 & 10 & 2 & 300 & 120 & 500000 \\
\hline $\mathrm{F}$ & 1 & 2600 & 10 & 2 & 300 & 60 & 300000 \\
\hline F & 2 & 2600 & 10 & 2 & 300 & 60 & 350000 \\
\hline $\mathrm{F}$ & 3 & 2600 & 10 & 2 & 300 & 60 & 400000 \\
\hline $\mathrm{F}$ & 4 & 2600 & 10 & 2 & 300 & 60 & 450000 \\
\hline $\mathrm{F}$ & 5 & 2600 & 10 & 2 & 300 & 60 & 500000 \\
\hline
\end{tabular}



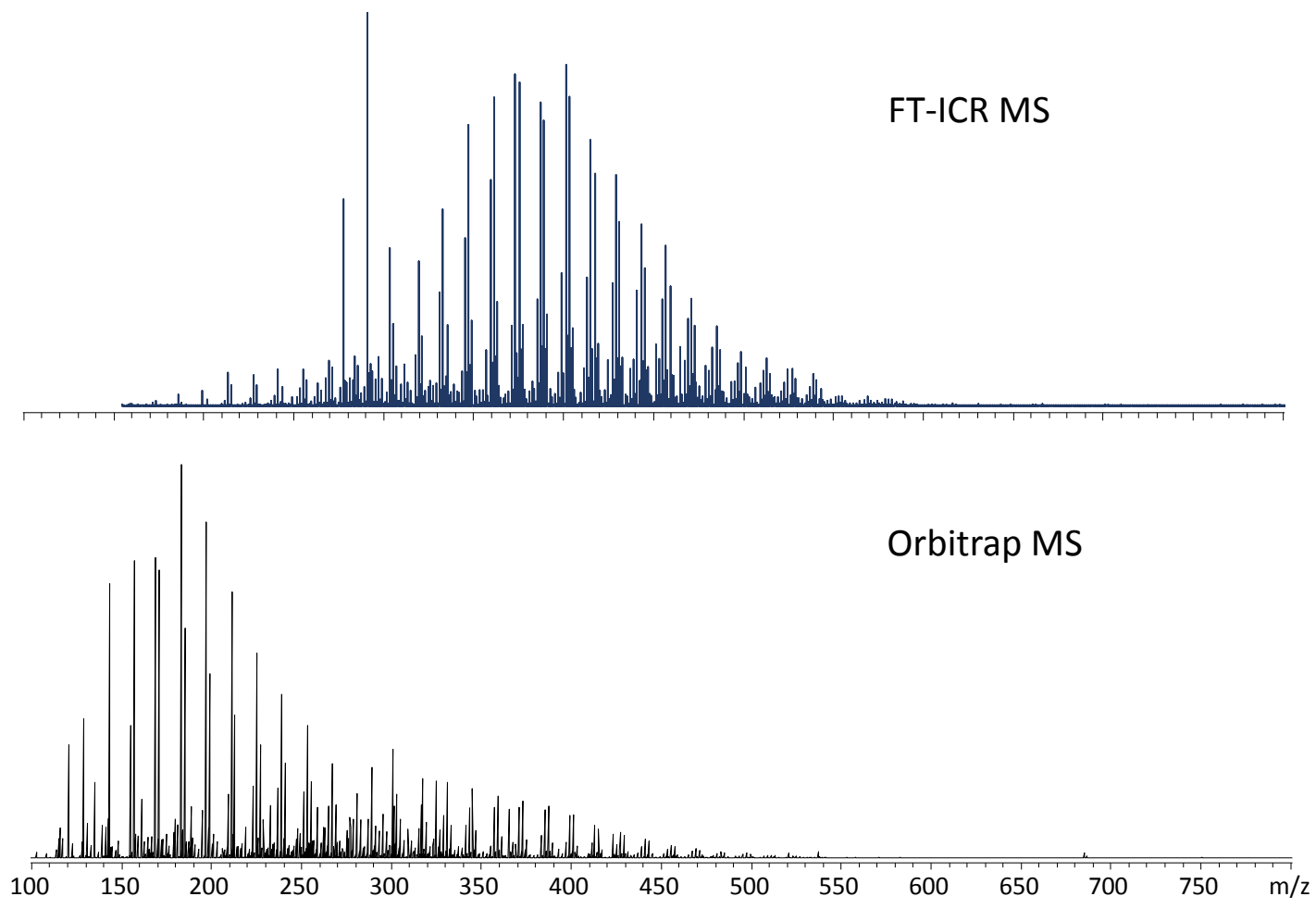

Figure S1. Mass distribution of condensate oil spectra of FT-ICR MS and Orbitrap MS
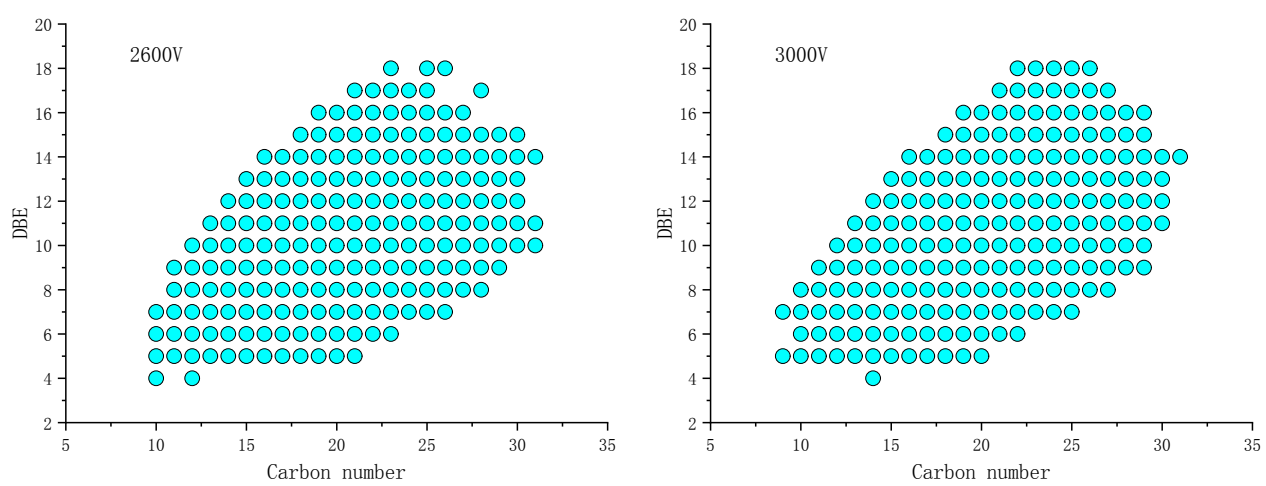

Figure S2. Distribution of O10 compounds under different ion spray voltage 


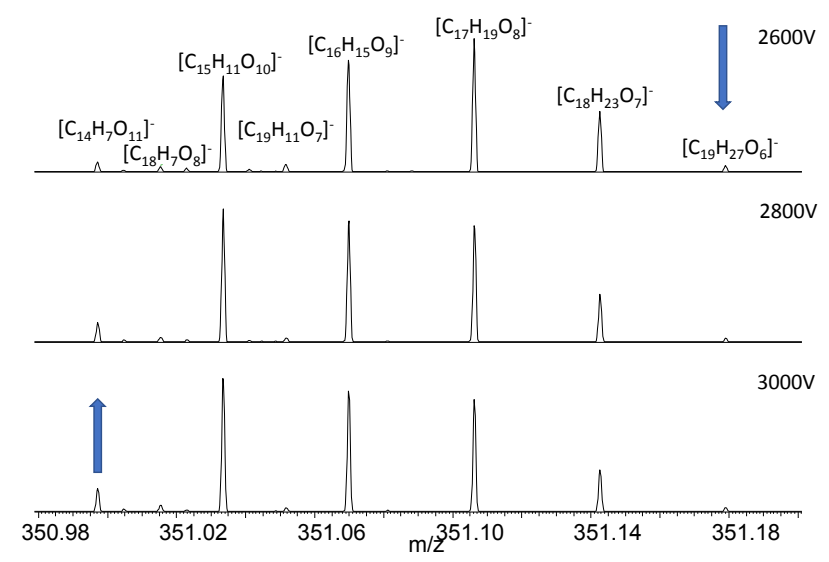

Figure S3. Expanded spectra under evaluated ion spray voltage at $\mathbf{m} / \mathbf{z} 351$
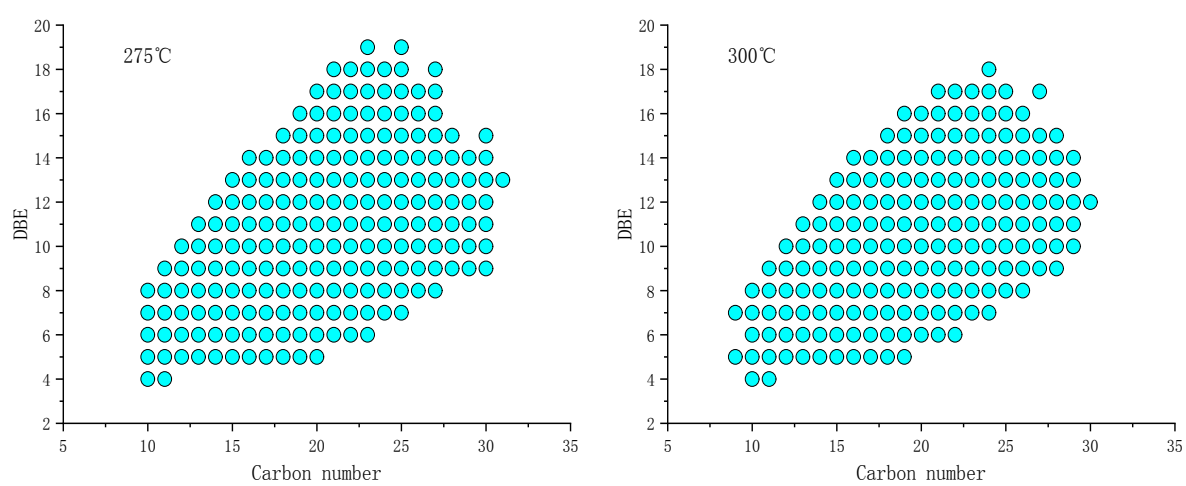

Figure S4. Distribution of 010 compounds under $275^{\circ} \mathrm{C}$ (left), $300^{\circ} \mathrm{C}$ (right) ion transfer tube temperature 

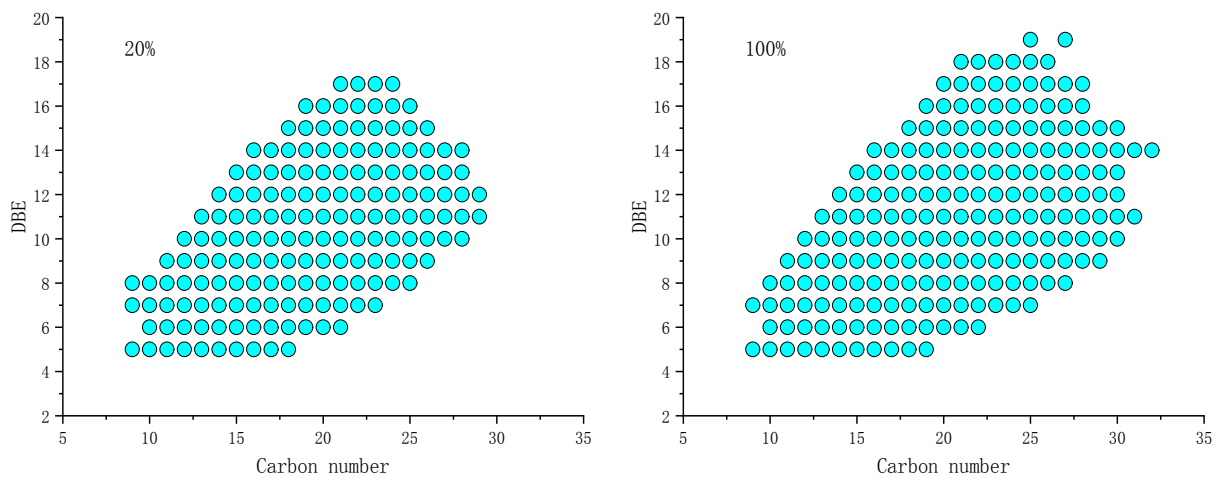

Figure S5. Distribution of 010 compounds under $20 \%$ (left), 100\% (right) rf lens

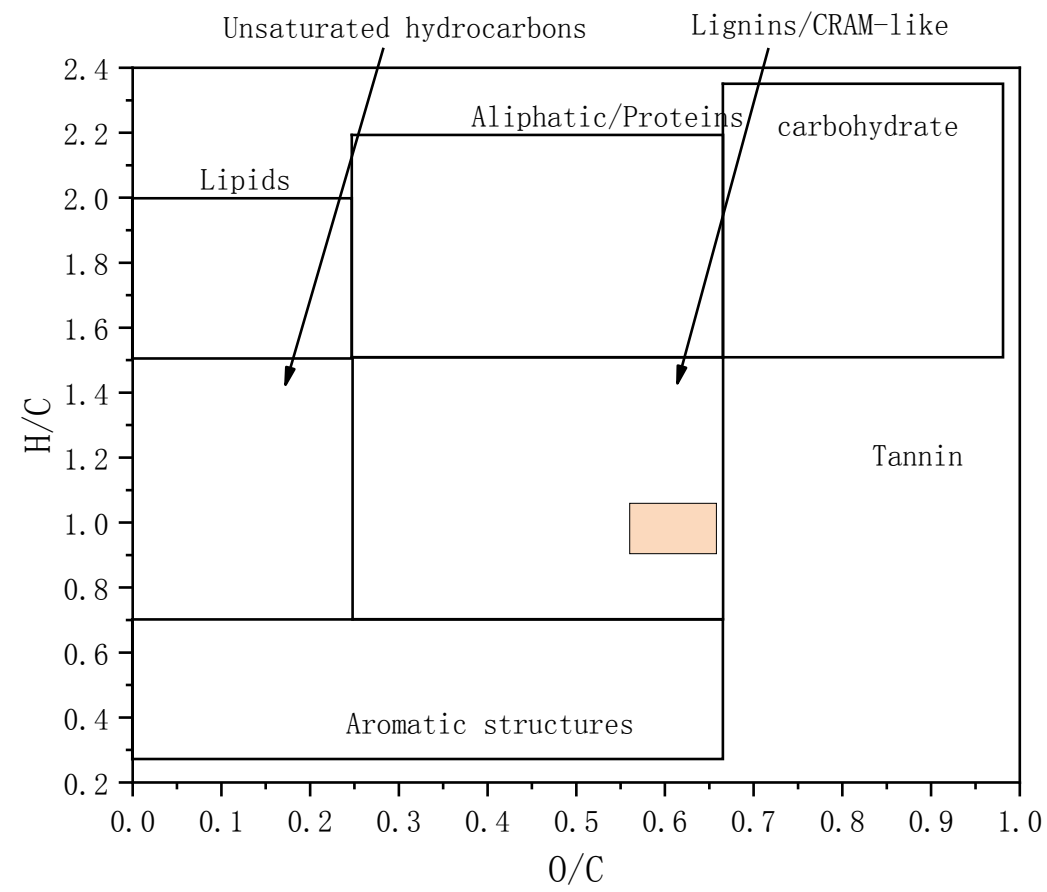

Figure S6. Typical category of compounds in different area of van Krevelen diagram 